Z. klin. Chem. u. klin. Biochem.

9. Jg., S. 136-142, März 1971

, 1

\title{
Eine neue Trennmethode für Radioimmunoassays von Proteohormonen: enzymatische Proteolyse des freien Hormons durch Pronase ${ }^{1}$ )
}

\author{
Von H. Wombacher und E. Nieschlag
}

\begin{abstract}
Aus der Abteilung fïr klinische Endokrinologie (Abteilungsvorsteber: Prof. Dr. C. Overzier) der II. Med. Klinik der Universität Mainz (Direktor: Prof. Dr. P. Scbölmericb)
\end{abstract}

(Eingegangen am 9. Oktober 1970)

Es wird eine neue Methode zur Trennung von freiem und an Antikörper gebundenem Hormon mitgeteilt. Die Trennung erfolgt dadurch, daß durch das Enzym Pronase die Hydrolyse des freien Hormons beschleunigt abläuft, während der Antikörper-Hormon-Komplex nicht angegriffen wird. Die Trennung wird exemplarisch an einem Radioimmunoassay für Insulin durchgeführt. Die Methode gestattet ein Arbeiten mit relativ niedriger spezifischer Aktivität des markierten Hormons und dem weichen Strahler ${ }^{125} \mathrm{~J}$, was von Wichtigkeit für schwierig zu markierende Proteo-Hormone ist. Grundsätzlich sollte die Methode der Trennung mit Pronase für die Radioimmunoassays kleinerer Proteohormone zu einer besonders für die Klinik geeigneten Methode werden. Denn die Handhabe ist einfach, schnell und genau.

\section{A new separation method for the radioin munoassay of proteobormones: enzymic proteolysis of the bormone with pronase}

A new method is reported for the separation of free and antibody-bound hormone. The separation is based on the enzymic proteolysis of the free proteohormone, while the antibody-bound hormone is not attacked. The radioimmunoassay of insulin is described as an example of the method, which permits the use of hormones of relatively low specific activity, labelled with soft emitter ${ }^{125} \mathrm{I}$. This is advantageous with proteohormones that are difficult to label. Principally this method of separation using pronase should be applicable to the specific microradioimmunoassay of many proteohormones, especially those of low molecular weight.

Die einzelnen Methoden zur tadioimmunologischen Bestimmung von Proteohormonen in Körperflüssigkeiten unterscheiden sich im wesentlichen durch die zur Trennung von freiem und an Antikörper gebundenem Hormon verwendeten Verfahren. Die zur Zeit bekannten Trennmethoden sind Elektrophorese $(1,2)$, Präzipitation des an Antikörper gebundenen Hormons durch einen zweiten Antikörper, die sog. „double antibody" Methode (3-6), oder die Adsorption des freien Hormons an eine feste Phase. In diesem Fall findet Aktivkohle (7), Ionenaustauscher (8) oder in der neuesten Version an Dextrangel gekoppelter Antikörper (9) Verwendung. Allen diesen Methoden ist eine spezifische Problematik eigen, und vielen Methoden steht zur Aufnahme in die klinische Routine großer zeitlicher und technischer Aufwand entgegen.

Dies hat uns veranlaßt, nach einer Trennmethode zu suchen, die in kurzer Zeit und mit einer StandardLaborausrüstung für die Klinik verläßliche Werte liefert. Als Ergebnis wird in der vorliegenden Arbeit eine Methode beschrieben, die auf der Trennung von freiem und an Antikörper gebundenem Hormon durch das Enzym Pronase beruht. Exemplarisch "wurde das neue Verfahren bei der radioimmunologischen Insulin-Bestimmung erprobt. Man darf aber mit Recht annehmen, daß sich das Verfahren auch bei anderen radioimmunologischen Bestimmungen bewähren wird.

\section{Material und Methodik}

Das zur Erstellung der Standardkurve eingesetzte Insulin ist Schweineinsulin. Der Antikörper ist in einem Anti-Schweineinsulin-Serum vom Meerschweinchen enthalten. Alle Präparate

1) Mit Unterstützung der Deutschen Forschungsgemeinschaft. sind lyophil getrocknet und mit Rinderalbumin stabilisiert. Das mit ${ }^{125} \mathrm{Jod}$ radioaktiv markierte Insulin stammt ebenfalls vom Schwein. Die spezifische Aktivität beträgt je nach Charge 100 bis $200 \mathrm{mC} / \mathrm{mg}$, die biologische Aktivität $27 \mathrm{E} / \mathrm{mg}$ vor der Jodierung (Bezugsquelle: Fa. Hoechst AG).

Das verwendete Rinderalbumin ist lyophil getrocknet und weist einen elektrophoretischen Reinheitsgrad von $100 \%$ auf.

Das Enzym Pronase (Protease aus Streptomyces griseus) ist gereinigt und lyophil getrocknet und wird von der Kaken Chemical Company, Tokio, bezogen. Die Aktivität des Enzyms beträgt 45000 PUK/mg.

Die radioaktive Messung exfolgte in einem $\gamma$-Spektrometer der Fa. Tracer-Lab.

\section{Lösungen}

Puffer

Als Puffex wird ein $0,05 \mathrm{M}$ Phosphatpuffer, der auf $\mathrm{pH} 7,4$ eingestellt ist, mit verschiedenen Albuminzusätzen verwendet. Der Puffer mit 1\% Albuminzusatz wird als Puffer I bezeichnet, der mit $0,25 \%$ Albuminzusatz als Puffer II und der Phosphatpuffer, der kein Albumin enthält, als Puffer III.

\section{Insulin-125 J-Stammlösung}

$2 \mathrm{mE}$ Insulin-125 $\mathrm{J}$ werden in $2 \mathrm{ml}$ bidest. Wasser gelöst und mit Phosphatpuffer II auf $10 \mathrm{ml}$ aufgefüllt. $(0,2 \mathrm{mE}$ Insulin_-125 J/ml).

\section{Reaktionslösung}

$7,5 \mathrm{ml}$ Insulin-125 J-Stammlösung werden in $250 \mathrm{ml}$ Phosphatpuffer II gelöst. Außerdem soll die Reaktionslösung das Antiserum in einer Verdünnung von 1:140000 enthalten. Die Herstellung der Reaktionslösung muß unter Eiskühlung erfolgen.

Insulin-125 J-Kontrollösung

$1,5 \mathrm{ml}$ Insulin-125 J-Stammlösung werden mit Phosphatpuffer II auf $50 \mathrm{~m} l$ aufgefüllt $(6 \mu \mathrm{E} / \mathrm{m} l)$.

\section{Insulinstandardlösung}

$10 \mathrm{mE}$ vom Schwein werdẹ ị $1 \mathrm{ml}$ bidest. Wasser aufgelöst und mit Phosphatpuffer I auf $25 \mathrm{ml}$ aufgefüllt $(400 \mu \mathrm{E} / \mathrm{m} /)$. Aus dieser Lösung wird mit Puffer I eine Verdünnungsreihe mit folgender Konzentration hergestellt: $2,4,6,8,12,20,28,40,80,160 \mu \mathrm{E} / \mathrm{ml}$. 
Tab. 1

Ansatzschema zur Aufstellung einer Standardkurve und zur Bestimmung des Insulins in den Analysenproben Probenummer: 1-3 Totalaktivität, 4-6 Kontrollwert, 7-29 Standardkurve, 30-31 Analysenprobenkontrollwert, 32 ff. Analysenproben

\begin{tabular}{|c|c|c|c|c|c|c|c|c|c|c|c|c|c|c|c|c|}
\hline Probenummer & $\underline{m}$ & i & i & T⿱一兀 & $\stackrel{n}{1}$ & $\frac{12}{ \pm}$ & $\frac{1}{b}$ & $\frac{a}{1}$ & $\begin{array}{c}\bar{N} \\
\text { ্ָ }\end{array}$ & 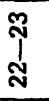 & ‡ั & ָิ & ঙ্ণী & pip & 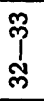 & 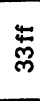 \\
\hline Insulinstandardlösung $[\mathrm{m} l]$ & 0 & 0 & $\mathbf{0}$ & 1 & 1 & 1 & 1 & 1 & 1 & 1 & 1 & 1 & 1 & 0 & 0 & 0 \\
\hline Phosphatpuffer I [ml] & 1 & 1 & 1 & 0 & 0 & 0 & 0 & 0 & 0 & 0 & 0 & 0 & 0 & 0 & 0 & 0 \\
\hline $\begin{array}{l}\text { Insulinkontrollösung }[\mathrm{ml}] \\
\text { (Insulin-123 J, } 6 \mu \mathrm{E} / \mathrm{ml} \text { ) }\end{array}$ & 0 & 1 & 0 & 0 & 0 & 0 & 0 & 0 & 0 & 0 & 0 & 0 & 0 & 1 & 0 & 1 \\
\hline $\begin{array}{l}\text { Reaktionslösung }[\mathrm{ml}] \\
\text { (Antikörper } 1: 140000 \text {, } \\
\text { Insulin-125 } \mathrm{J}, 6 \mu \mathrm{E} / \mathrm{ml} \text { ) }\end{array}$ & 1 & 0 & 1 & 1 & 1 & 1 & 1 & 1 & 1 & 1 & 1 & 1 & 1 & 0 & 1 & 0 \\
\hline $\operatorname{Serum}(1: 5)[\mathrm{ml}]$ & 0 & 0 & 0 & 0 & 0 & 0 & 0 & 0 & 0 & 0 & 0 & 0 & 0 & 1 & 1 & 1 \\
\hline \multicolumn{17}{|c|}{ Inkubation bei $4^{\circ} 54 \mathrm{Stdn}$} \\
\hline Pronase $(350 \mu \mathrm{g} / \mathrm{ml} l)[\mathrm{m} l]$ & 0 & 1 & 1 & 1 & 1 & 1 & 1 & 1 & 1 & 1 & 1 & 1 & 1 & 1 & 1 & 1 \\
\hline \multicolumn{17}{|c|}{ Inkubationszeit $30 \mathrm{Min}$. bei $37^{\circ}$ (öfter umschütteln) } \\
\hline $\begin{array}{l}\text { Trichloressigsäure [ml] } \\
\text { (20proz.) }\end{array}$ & 0 & 2 & 2 & 2 & 2 & 2 & 2 & 2 & 2 & 2 & 2 & 2 & 2 & 2 & 2 & 2 \\
\hline
\end{tabular}

Abzentrifugieren bei $900 \mathrm{~g}$ für $20 \mathrm{Min}$. Abdekantieren des Öberstandes und Messung des Rückstandes

\section{Enzj'mlösung}

$35 \mathrm{mg}$ lyophilisierte Pronase wird in $100 \mathrm{~m} /$ Phosphatpuffer III unter Rühren gelöst bzw. suspendiert. Die Enzymlösung wird jeweils frisch bereitet.

\section{Tricbloressigsäurelösung}

20proz. wäßr. Lösung, die vor Gebrauch auf Eisschranktemperatur gekühlt wird.

\section{Serumverdiinnung}

Das Serum wird mit Puffer III 1:5 verdünnt.

(Die Verdünnung soll mindestens 1:5 betragen. Bei Kontrollwerten, die mehr als $10 \%$ der Gesamtradioaktivität zeigen, muß das Serum evtl. höher verdünnt werden. Die Höhe der Verdünnung ist durch die Nachweisgrenze der Methode beschränkt.)

\section{Arbeitsgang}

In Tabelle 1 ist der Arbeitsgang mit den einzelnen Pipettiervorgängen dargestellt.

\section{Auswertungsverfahren}

Man erhält die Standardkurve dadurch, daß das Verhältnis von an Antikörper gebundenem Insulin (B) zu freiem Insulin (F), als $B / F$ bezeichnet, in Abhängigkeit von den zugesetzten Konzentrationen an freiem Hormon aufgetragen wird. Die unbekannten Insulinkonzentrationen der Analysenproben werden ermittelt, indem man das bei ihnen ermittelte B/F Verhältnis mit der Standardkurve vergleicht.

Das Verhältnis B/F für die einzelnen Werte der Standardkurve wird wie folgt ermittelt:

Es bedeuten,

$\mathrm{TA}=$ Totalaktivität (Probe 1-3) Impulsrate der nicht der enzymatischen Hydrolyse unterzogenen Proben 1-3.

$\mathrm{N}=$ Zählrate (Imp./Min.) des nach dem Abdekantieren verbleibenden Rückstandes der Proben 4- 6 (nichtspezif. Bindung des Hormons in Abwesenheit des Antikörpers und sonstiges nicht hydrolysiertes radioaktiv markiertes Hormon). $\mathrm{N}$ soll nicht über $10 \%$ der TA ansteigen.

$X_{i}=$ Impulsrate (Imp./Min.) des Rückstandes nach enzymatischer Hydrolyse und dem Dekantieren der Standardproben 7-29.

$B=X_{1}-N$ (an Antikörper gebundenes Hormon)

$\mathrm{F}=\mathrm{TA}-\mathrm{X}_{\mathrm{i}}$ (freies nicht an Antikörper gebundenes Hormon) $\frac{B}{F}=\frac{X_{1}-N}{T A-X_{1}}$.

Bei der Ermittlung der unbekannten Analysenproben wird prinzipiell in der gleichen Weise vorgegangen. Es bedeutet hier im Unterschied zu oben $\mathbf{X}_{1}=$ Impulsrate (Imp./Min.) des Rückstandes der Analysenproben nach enzymatischer Hydrolyse und Abdekantieren.
$N=$ Impulsrate (Imp./Min.) des Rückstandes der Analysenkontrolle nach enzymatischer Hydrolyse und Abdekantieren.

Die zu den jewcils gefundenen B/F Werten gehörigen Abszissenwerte liefern die gesuchte Insulinkonzentration.

Die Ermittlung des Quotienten B/F geht auf BERSON und Yalow (10) zurück.

Prinzip der Trennung

Nach beendigter Gleichgewichtseinstellung der Antigen-Antikörper-Reaktion erfolgt die Trennung von freiem und an Antikörper gebundenem Hormon durch enzymatische Hydrolyse. Das freie Hormon wird rasch hydrolysiert, während das an den Antikörper gebundene Hormon gegen die enzymatische Hydrolyse geschützt ist. Im Prinzip läßt sich das folgendermaßen darstellen:

$\mathrm{AKH} r+\mathrm{AKH}+\mathrm{H}^{\mathrm{r}}+\mathrm{H} \underset{\mathrm{K}_{\mathrm{H}}}{\stackrel{\text { Pronase }}{\rightleftharpoons}} \mathrm{AKH}^{\mathrm{r}}+\mathrm{AKH}+\mathrm{P}$

$\mathrm{AKH}^{\mathrm{r}}=$ Antikörper-Hormon-Komplex (Hormon radioaktiv markiert)

$\mathrm{AKH}=$ Antikörper-Hormon-Komplex

$\mathrm{H}^{\mathrm{r}} \quad=$ radioaktiv markiertes Hormon

$\mathrm{H}=$ Hormon

$\mathrm{P} \quad=$ Hydrolyseprodukte

$\mathrm{K}_{\mathrm{H}}=$ Gleichgewichtskonstante der Hydrolysereaktion.

Die Enzymreaktion wird dann mit Trichloressigsäure (20proz.) abgestoppt. Dabei bleiben die durch die Proteolyse des Enzyms Pronase entstandenen nicdermolekularen Hydrolyseprodukte in Lösung, während alle höhermolekularen Eiweiße, darunter auch der Antikörper-Hormonkomplex, ausfallen. Diese können dann durch Zentrifugation abgetrennt werden.

\section{Ergebnisse}

Die Trennung von freiem und gebundenem Hormon durch Hydrolyse nuit dem Enzym Pronase führt zu dem in der folgenden Standardkurve dargestellten Ergebnis (Abb. 1).

Die Präzision mit der das Verhältnis $\mathrm{B} / \mathrm{F}$ gefunden wird, kann durch die Standardabweichung beschrieben werden. Nach einer von KaISER und Specker (11) angegebenen Formel läßt sich die Standardabweichung folgendermaßen berechnen:

$$
s=\sqrt{\frac{\frac{\sum_{1}^{n} \sigma_{1}^{2}}{N-g}}{N-g}}
$$




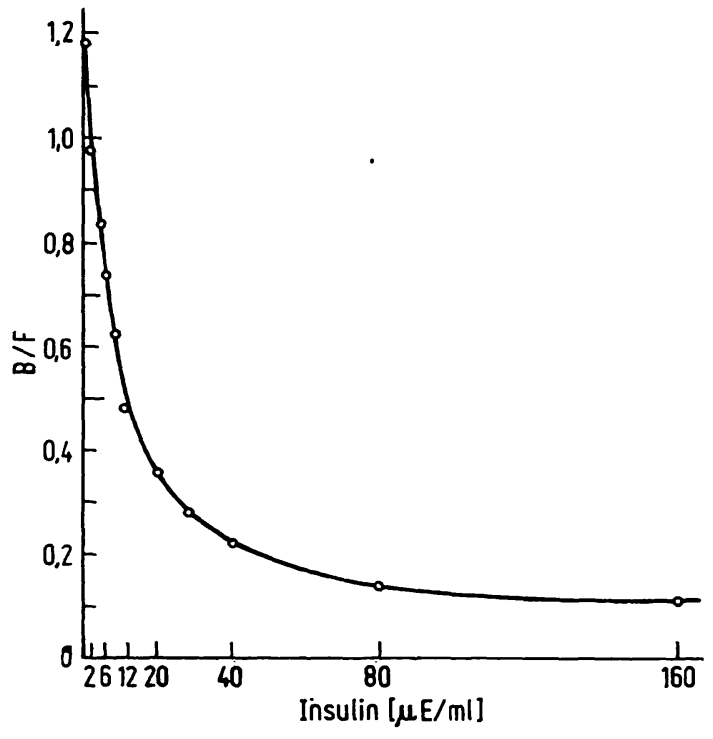

Abb. 1

In der Standardkurve ist das Verhältnis B/F (Verhältnis des an Antikörper gebundenen Hormons zu freiem Hormon) als Funktion der Konzentration des nicht markierten Hormons aufgetragen

Die Formel gilt für den Fall, daß $n$ Messungen in $g$ Meßgruppen vorliegen. Innerhalb jeder Gruppe wird der Mittelwert gebildet und die zu dem jeweiligen Mittelwert gehörigen Abweichungsquadrate der einzelnen Meßwerte. Die $\sigma^{2}$-Werte werden dann ohne Rücksicht auf die Tatsache, daß sie aus verschiedenen Meßgruppen stammen, addiert. Die so gefundene Standardabweichung stellt einen Näherungswert dar. Um über den wahren Fehler eine statistisch gesicherte Aussage machen zu können, wird nach der $\mathrm{t}$-Verteilung s modifiziert. Für eine Sicherheit von $99 \%$ und bei 54 Messungen ergibt sich für den Faktor $t$ der Wert 2,67 mit dem die Standardabweichung zu multiplizieren ist. Auf diese Weise ergibt sich eine Standardabweichung von 0,0059 .

Sicherstellung von Unterschieden (nach Katser und Specker (11))

Wie man anhand der Standardabweichung sieht, kann der Unterschied der Meßwerte der zur Aufstellung der Standardkurve eingesetzten Konzentrationen in sehr hohem Maße als gesichert gelten. Denn in jedem Fall ist die Differenz der Meßwerte zweier verschiedener Konzentrationen größer als die Standardabweichung für $\mathrm{p} \leqq 0,001$.

$$
\mathrm{B} / \mathrm{F}_{1}-\mathrm{B} / \mathrm{F}_{2}>\mathrm{s} \cdot \mathrm{t}(\mathrm{N})(\mathrm{S})=0,00769(\mathrm{t}=3,48)
$$

Bestimmung der Nachweisgrenze

Für die Nullwerte wurde die Standardabweichung aus 10 Messungen mit 0,0278 separat bestimmt, weil die Nullwerte größeren Schwankungen unterworfen sind als die übrigen Werte. Für den B/F Wert, der für die niedrige Konzentration $2 \mu \mathrm{E} / \mathrm{m} l$ Insulin gemessen wird, zeigt sich ein signifikanter Unterschied zu dem Nullwert. Diese signifikante Differenz ergibt sich aus der folgenden Gleichung, durch welche die Nachweisgrenze definiert ist (KAISER und SPECKER (11)).

$$
\begin{array}{lll}
\mathrm{B} / \mathrm{F}_{\mathbf{x}}-\mathrm{B} / \mathrm{F}_{\mathrm{o}}>\mathrm{s}: \mathrm{t} & \\
& 0.0278 \cdot 3.17, \quad \mathrm{t}=3,17 \\
& 0.0911 & \mathrm{p} \leqq 0,01 \\
& & \mathrm{~N}=10 \\
\mathrm{~B} / \mathrm{F}_{\mathbf{x}} & \left\langle\mathrm{B} / \mathrm{F}_{\mathrm{o}}-(\mathrm{s} \cdot \mathrm{t})\right. & \\
& 1,1837-0,0911 \\
\mathrm{~B} / \mathrm{F}_{\mathbf{x}} & \langle 1,0926 &
\end{array}
$$

$B / F_{x}=$ sicher von Null verschiedener Wert

$\mathrm{B} / \mathrm{F}_{\mathbf{0}}=$ Mittelwerte der Nullwerte

$t=$ von der angelegten Sicherheit $(p \leqq 0,01)$ und der Anzahl der Messungen abhängiger Faktor mit dem die Standardabweichung nach der Student-Verteilung korrigiert werden muß, um Aufschluß über den wahren Fehler zu erhalten.

Die Wiederauffindung von eingesetztem Hormon ist in Abbildung 2 dargestellt. Für dieses Experiment wurde als insulinfreies Serum der Puffer I verwendet, der mit verschiedenen Insulinkonzentrationen versetzt wurde und dann entsprechend dem Vorgehen beim Serum bzw. Plasma verdünnt wurde.

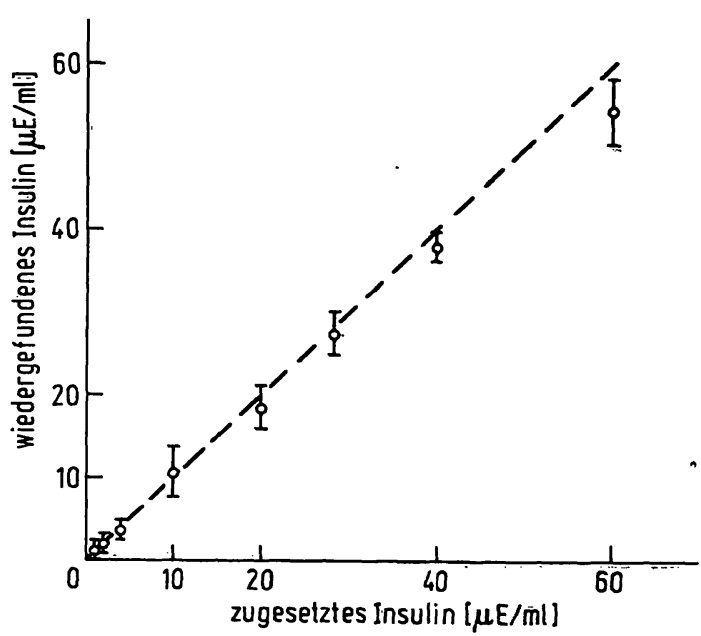

Abb. 2

In dieser Darstellung sind die gefundenen Werte in Abhängigkeit von der zugesetzten Menge Insulin aufgetragen. Idealerweise sollten alle Werte auf der für $100 \%$ Wiederauffindung eingezeichneten Geraden liegen. Die eingezeichneten Werte sind Mittelwerte aus 10 Bestimmungen mit der zugehörigen Standardabweichung (korr. nach der $t$-Verteilung für eine Sicherheit von $99 \%$ )

\section{Insulinnüchternwerte von Normalpatienten}

Der bei 8 Normalpersonen gefundene Durchschnittswert der Insulinkonzentration im Plasma im Nüchternzustand beträgt $16 \mu \mathrm{E} / \mathrm{m} l$. Der Bereich der gefundenen Werte erstreckt sich von $0-33 \mu \mathrm{E} / \mathrm{m} l$. Dabei ist $\mathrm{zu}$ berücksichtigen, daß das Serum eine Verdünnung von 1:5 erfährt und deshalb infolge der Empfindlichkeitsgrenze der Methode Werte unter $10 \mu \mathrm{E} / \mathrm{m} l$ nicht sicher von Null zu unterscheiden sind.

\section{Diskussion}

Idealerweise sollte die Trennung von freiem und an Antikörper gebundenem Hormon vollständig und augenblicklich erfolgen. Es gibt keine Methode, die 
diese theoretische Forderung vollständig erfüllt. Die vorliegende Methode, die auf einer beschleunigten Hydrolyse des freien, nicht gebundenen Hormons durch das Enzym Pronase beruht, erfüllt die oben gestellteForderung in dem $\mathrm{Maße}$, daß in geeigneten Konzentrationsverhältnissen und in einer geeigneten Zeit der größte Teil des nicht an Antikörper gebundenen Hormons hydrolysiert ist; das an Antikörper gebundene Hormon aber so gut wie nicht angegriffen ist.

Es steht außer Frage, daß nicht beliebige, proteolytisch wirksame Enzyme verwendet werden können. Es ist sogar so, daß die meisten proteolytisch wirksamen Enzyme z. B. Papain, Trypsin, Chymotrypsin oder Pepsin, sich nicht als geeignet erwiesen haben. Auch das von MrTCHELl und Mitarbeitern $(12,13)$ vorgeschlagene Ficin hält keinen Vergleich mit Pronase aus.

Es ist zweifelsohne eine wichtige und aufschlußreiche Untersuchung von BERSON und YALOW (14) gewesen, in der sie feststellten, daß Insulin einen Schutz gegen den proteolytischen Einfluß der Insulinase durch die Bindung an einen spezifischen Antikörper erhält. Es wäre interessant zu erfahren, ob die Antikörper für die Proteohormone generell solche Schutzfunktion besitzen. Arbeiten in dieser Richtung fehlen. Andere, Insulin in geringerem Maße bindende Eiweiße, wie z. B. Albumin besitzen eine nur sehr schwach ausgeprägte Schutzfunktion (vgl. Wirliams (15)).

Die geeigneten Bedingungen für die enzymatische Hydrolyse werden entsprechend den Angaben von Noмото und Mitarbeitern (16) so gewählt, daß der $\mathrm{pH}$ Wert, die Ionenstärke und die Temperatur im Optimum der Enzymhydrolyse liegen. Eine weitere Erhöhung der Temperatur, die die enzymatische Hydrolyse ohne Nachteil für die Funktion des Enzyms Pronase steigern würde, wird mit Rücksicht auf die dann auch gesteigerte Dissoziation des Antikörper-Insulin-Komplexes und einer möglichen Denaturierung vermieden. So ist die beobachtete, im Vergleich etwa zur Ionenaustausch-Methode (8), scheinbar niedrigere prozentuale Bindung von markiertem Hormon an einen identischen Antikörper, damit erklärbar, daß die Inkubation bei $37^{\circ}$ zur enzymatischen Hydrolyse die Dissoziationskonstante des Antikörper-Hormon-Komplexes ansteigen läßt, bzw. die Assoziationskonstante heruntersetzt. Aus der gesteigerten Dissoziationsreaktion des Antikörper-Hormon-Komplexes während der Inkubationszeit bei $37^{\circ}$ ergibt sich die Forderung, die Zeit für die enzymatische Hydrolyse so kurz wie möglich zu halten. Mit der eingesetzten Pronase findet ein Enzym Verwendung, das über die höchste proteolytische Aktivität aller bisher bekannten Enzyme verfügt. Die Pronase ist insofern hervorragend geeignet, die Inkubationszeit für die enzymatische Hydrolyse des freien Hormons auf ein Mindestmaß zu beschränken.

Die Frage allerdings, warum das freie Hormon nahezu selektiv hydrolysiert wird und der Antikörper-HormonKomplex dagegen nur einer geringen, unter geeigneten Bedingungen überhaupt nicht ins Gewicht fallenden Hydrolyse unterliegt, diese Frage kann zunächst nicht beantwortet werden. Die für die Pronase festgestellte geringe Substratspezifität (Noмото und Mitarbeiter (16)) macht dieBeantwortung dieser Frage noch schwieriger.

Die Pronase, dic eine Peptidase neueren Typs darstellt, besitzt sowohl die Eigenschaften vieler Endo- wie Exopeptidasen. Sie läßt sich nach den klassischen Einteilungsprinzipien weder als Endo- noch als Exopeptidase charakterisieren. Das viclleicht hervorstehendste Merkmal ist ihre Fähigkeit, auch sehr niedermolekulare Eiweiße zu hydrolysieren. So werden Oligopeptide, ja sogar Tri- und Dipeptide zu den einzelnen Aminosäuren hydrolysiert (Nomoro und Mitarbeiter (16)). Trotz der Kenntnisse über die kinetischen Eigenschaften der Pronase, die Nомото und Mitarbeiter (16) und Hasimimoro und Mitarbeiter (17) geliefert haben, bleibt es unklar, warum sich freies und an Antikörper gebundenes Hormon auf die beschricbene Weise so gut trennen lassen, beziehungsweise wieso der Antikörper, der selber zu den Eiweißen zu rechnen ist, eine solche Schutzfunktion für das Hormon auszuüben vermag. Weitere kinetische Untersuchungen, die diesen Sachverhalt erhellen sollen, werden zur Zeit von uns vorgenommen, denn wir sind der Ansicht, daß dieser Frage allgemeine Bedeutung zukommt.

Es kommt der enzymatischen Trennung von freiem und an Antikörper gebundenem Hormon sehr zustatten, daß die für die Inkubation des Antikörpers mit markiertem und unmarkiertem Hormon gewählten Bedingungen ( $p H$ und Ionenstärke) auch für die anschließende enzymatische Hydrolyse mit Pronase im Optimum liegen. Falls einmal die enzymatische Trennung durch einen im Serum befindlichen Hemmstoff des Enzyms (evtl. Medikamente) gestört sein sollte, dann ist dies an dem Kontrollwert des Serums zu erkennen. Der beispielsweise bei der Trennung mit Ionenaustauschern (z. B. Amberlite CG 400) zu beobachtende Effekt einer unter Umständen unvollständigen Trennung infolge der Anwesenheit zu großer Heparinmengen (Blutabnahme) ist bei der Trennung durch das Enzym Pronase nicht vorhanden. Auch stören die evtl. bei einer Schilddrüsentherapie verabreichten Thyroxingaben im Gegensatz zur Ionenaustausch-Methode (8) die Trennung von freiem und gebundenem Hormon nicht. Grundsätzlich können bei der Ionenaustausch-Methode (8) alle Stoffe mit polarem bzw. anionischem Charakter als Störfaktor in Frage kommen. Dagegen konnten wir bei der Trennung mit Pronase bisher keinen Hemmstoff finden. Gegenüber der Trennung mit einem zweiten Antikörper hat die Trennung mit Pronase den Vorteil, daß für den zweiten Antikörper die zusätzliche Inkubationszeit entfällt, die die Untersuchung in der Regel um zwei Tage verlängert; nämlich um die Inkubationszeit, die der zweite Antikörper zur Einstellung des Gleichgewichtes benötigt. Die Trennung mittels Papierchromatogramm-Elektrophorese liefert zwar nach Mitteilung von YALOW und BERSON (18) recht gute Ergebnisse, verlangt aber einen für klinische Verhältnisse hohen Aufwand und sctzt eine radioaktive Insulin-Präpa- 
ration von hoher spezifischer Aktivität voraus. Die sogenannte "solid phase" Methode (9, 19), die in letzter Zeit starke Beachtung findet, erfährt ihre eingeschränkte Anwendbarkeit durch die mit recht hohen experimentellen Schwierigkeiten behaftete Kupplung von Antikörper an Isothiocyanatophenoxyhydroxypropyl-Sephadex (9) oder an Polytetrafluoräthylen- $\gamma$ aminostyren (19) und durch die Standardisierung der gewonnenen Produkte. Auch die von CATt und Tregear (20) entwickelte Technik mit den „coated test tubes" ist weit aufwendiger als die beschriebene Trennung mit Pronase. Die jüngste Arbeit mit dieser Technik (Ceska und Mitarbeiter (21)) zeigt, daß die Reproduzierbarkeit dieser Methode geringer ist als bei der obengenannten "solid phase" Methode und daß sie in ihrer allgemeinen Anwendbarkeit doch recht großen Einschränkungen unterliegt. Vergleichbar in Schnelligkeit und Einfachheit der Durchführung mit der Pronase-Trennmethode sind die Methoden, die mit Ionenaustauscher (8) oder mit ",coated charcoal“ (7) arbeiten. Nachteilig wirken sich jedoch bei diesen Methoden unspezifische Adsorptionseffekte aus.

Die zur statistischen Beurteilung der Pronase-Trennmethode verwendeten Begriffe wie "Genauigkeit", „Verläßlichkeit" und „Empfindlichkeit" sind von verschiedenen Autoren verschieden definiert. Es herrscht, auch bedingt durch die Problematik, die in den Begriffen steckt, kein einheitlicher Sprachgebrauch. Beispielsweise sollte der Empfindlichkeitsbegriff gerade im Fall der Radioimmunoassays mehr als nur die Nachweisgrenze beinhalten, oder aber beide Begriffe sollten getrennt von einander definiert werden (vgl. BERson und Yalow (22), EkrNs (23)). Die im vorliegenden Fall verwendeten Kriterien, die nach einem Vorschlag von KAISER und SPECKER (11) benutzt werden, sollen mehr dazu dienen, die Möglichkeit einer sicheren und effektiven Trennung von freiem und an Antikörper gebundenem Hormon durch das Enzym Pronase zu beschreiben, als eine vollständige Charakterisierung des vorliegenden Radioimmunoassays zu liefern. Dieses einfachere Verfahren erscheint vor allem deswegen gerechtfertigt, weil Spezifität, Verläßlichkeit, Genauigkeit und Empfindlichkeit in erster Linie von den eingesetzten Substanzen abhängen und erst in zweiter Linie von der Güte der Trennung. So ist z. B. die Güte des Antikörpers, d. h. seine Spezifität und seine Avidität (die oft angegebene Verdünnung des eingesetzten Antiserums ist keine sichere Information), ganz entscheidend für die Empfindlichkeit und Genauigkeit der Methode. Ebenso spielt die Höhe der spezifischen Aktivität des markierten Hormons, seine Konzentration im Verhältnis zur Konzentration des Antikörpers im Ansatz, seine Reinheit und auch seine Identität mit dem nachzuweisenden Hormon eine wesentliche Rolle.

Die letztgenannten Gesichtspunkte sind auch für das zur Aufstellung der Standardkurve verwendete, nichtmarkierte Hormon von Bedeutung (vgl. (1), (22)). Insofern sind die erwähnten Verläßlichkeitskriterien schon durch die verwendeten Substanzen, wie sie unter Material und Methoden angegeben sind, festgelegt.

Die von uns als Genauigkeítskriterium ermittelte Standardabweichung erfolgte rein numerisch aus den Werten, die zur Aufstellung der Standardkurve führten. Bei der Darstellung der Wiederauffindung sollte man bedenken, daß bei der Auswertung von Proben unbekannten Gehalts eine mit weiteren. Fehlern behaftete Ablesung von der Standardkurve notwendig ist, was eine Vergrößerung der Standardabweichung bei der Bestimmung von Proben unbekannten Gehalts zur Folge hat. Wie man weiß; liefert ein Radioimmunoassay eine nicht lineare Dosis-Wirkungskurve, wenn das Verhältnis $\mathrm{B} / \mathrm{F}$ gegen bekannte Mengen von nichtmarkiertem Hormon aufgetragen wird. Infolge der Nichtlinearität der Funktion (Potrs und Mitarbeiter (24), BERson und YaLOW (10), EKINS und Mitarbeiter (25)) ist eine routinemäßige Interpolation über den ganzen Bereich der Standardkurve schwierig und nicht exakt möglich. Eine Beschränkung der Interpolation auf einen schmalen linearen Bereich der Kurve würde den auswertbaren Bereich des Assays merklich einschränken. Von verschiedenen Autoren wurden deshalb Vorschläge zur Linearisierung dieser gewöhnlich bei den Radioimmunoassays auftretenden Funktion gemacht, um eine schnellere und exaktere Kalkulation der Ergebnisse zu ermöglichen.

Oft ist eine Linearisierung durch semi-logarithmische Auftragung möglich. Hunrer $(26,27)$ beispielsweise verwendet sie zur Darstellung der Meßergebnisse der Standardkurve und zur Auswertung der Analysenergebnisse. Halb- und doppelt-logarithmische Auftragungen werden auch von HaLES und RandLE (5), Quabbe (28) und MeLANI und Mitarbeitern (2) verwendet. Nach einem Vorschlag von GadDum $(29,30)$ können solche Auftragungen zur Bestimmung eines Genauigkeitskriteriums bei Bioassays dienen. Darauf zurückgehend werden bei den letztgenannten Autoren solche Auftragungen auch in entsprechender Weise zur Bestimmung eines Genauigkeitskriteriums bei den Radioimmunoassays herangezogen. Dieses Vorgehen $\mathrm{muß}$ aber mit einiger Skepsis betrachtet werden, denn je nach Assay gelingen die Linearisierungen gut oder weniger gut, ohne daß dadurch unmittelbar etwas über die verschiedene Genauigkeit der einzelnen Assays sich aussagen ließe. So findet beispielsweise QUABBE (28) bei ein und demselben Radioimmunoassay verschiedene Werte für $\lambda, \mathrm{d}$. h. für die Genauigkeit, $a b-$ hängig davon, ob aus einer halb- oder doppeltlogarithmischen Auftragung bestimmt wird. Unserer Meinung nach ist die Bestimmung von $\lambda$, die bei den Bioassays ihre Berechtigung haben mag, als entscheidendes Genauigkeitskriterium zum Vergleich der einzelnen Radioimmunoasșays untereinander nur mit großen Einschränkungen möglich. Im vorliegenden Fall liefert die halblogarithmische Auftragung (Abb. 3), wie man sieht, keine gute Linearität.

Es zeigt sich vielmehr in dieser Transformation ein s-förmiger Verlauf der Kurve. Dies weist darauf hin, 

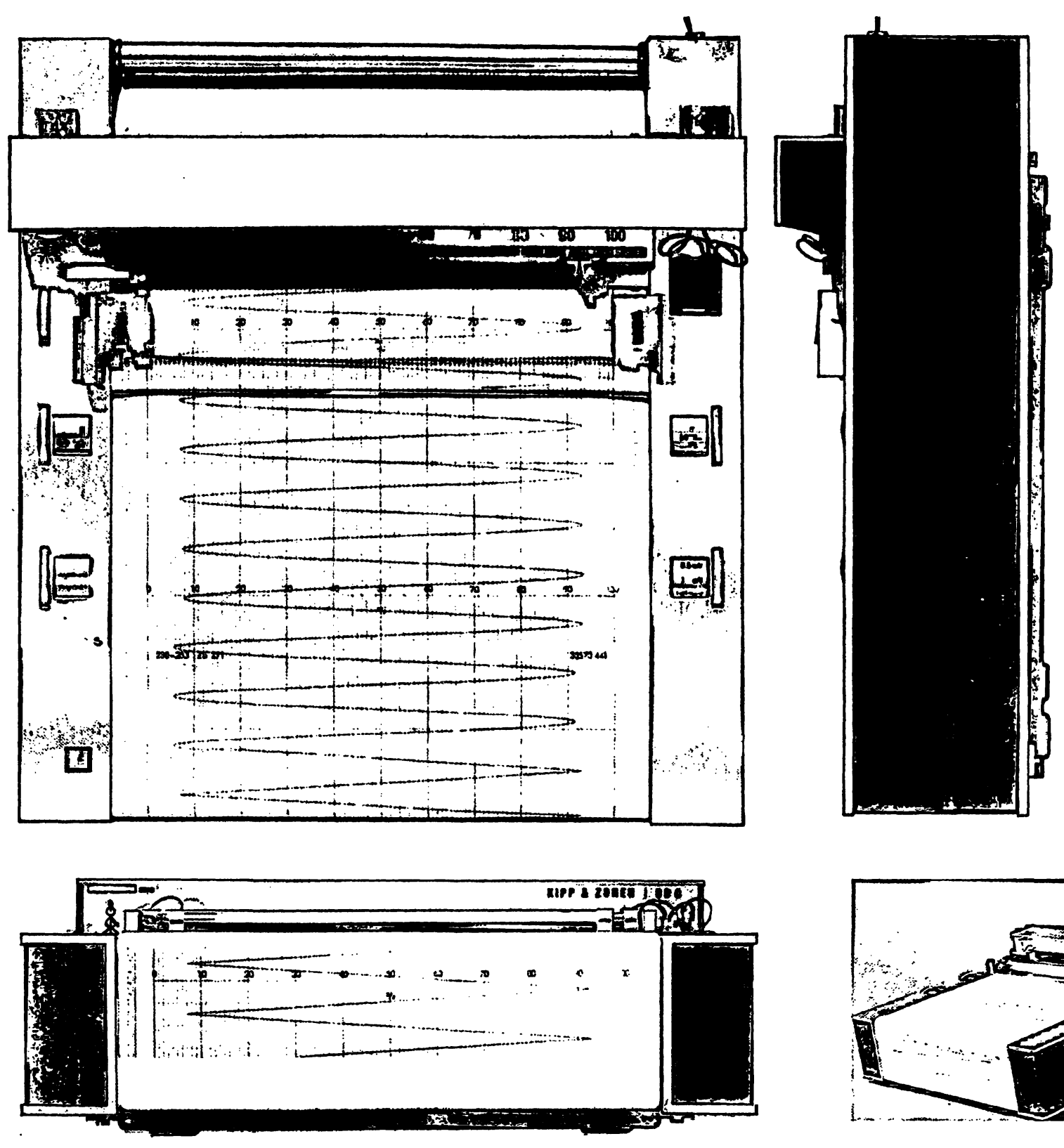

\section{Sie brauchen Kipp wenn Sie messen und registrieren}

\section{RIPP\&ZONEN}

6242 Schönberg/Ts. Wiesenau 5 Telefon 06173-5071 


\section{FRACPAC LB 9300}

Automatischer gekühlter

Fraktionssammler für 240 Proben

als Tischgerät (ca. $60 \times 60 \mathrm{~cm}$ )

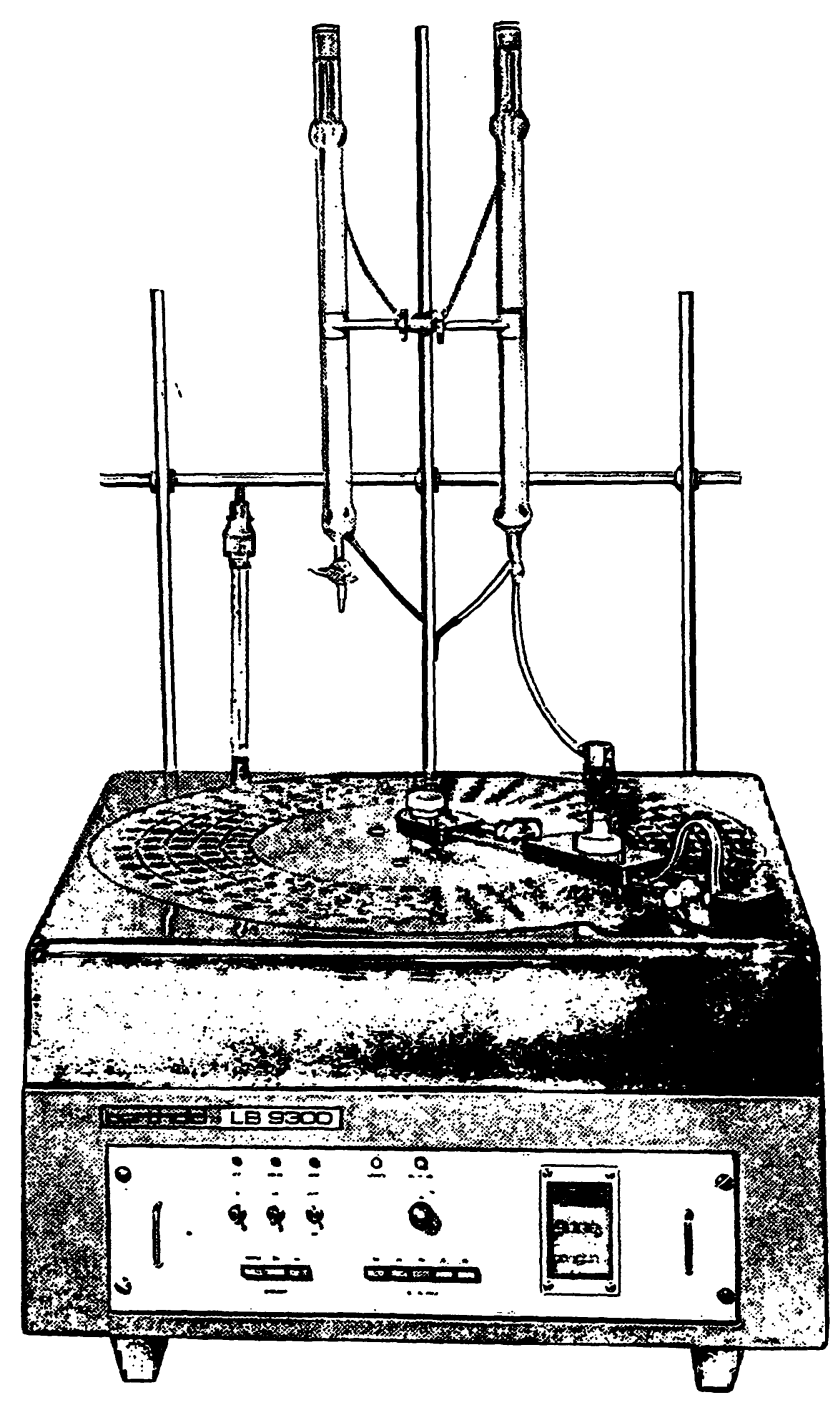

Temperatureinstellung

für Proben und Trennsäulen

von +2 bis $+50^{\circ} \mathrm{C}$

Vorwahl von Zeit oder Tropfenzahl

\section{Vertriebs-GmbH für Meßtechnik}

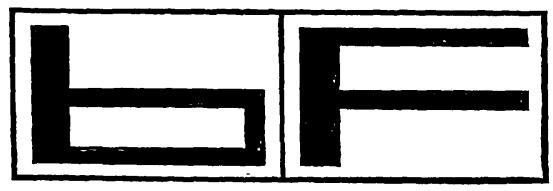

75 Karlsruhe-

Durlach

Bergwaldstr. 30

Tel. 0721/401011
Sie ist vielseitiger geworden, die "leise" Schüttelmaschine W-2! Jetzt ist Bewegung da für viele Scheidetrichter und noch mehr Reagenzgläser

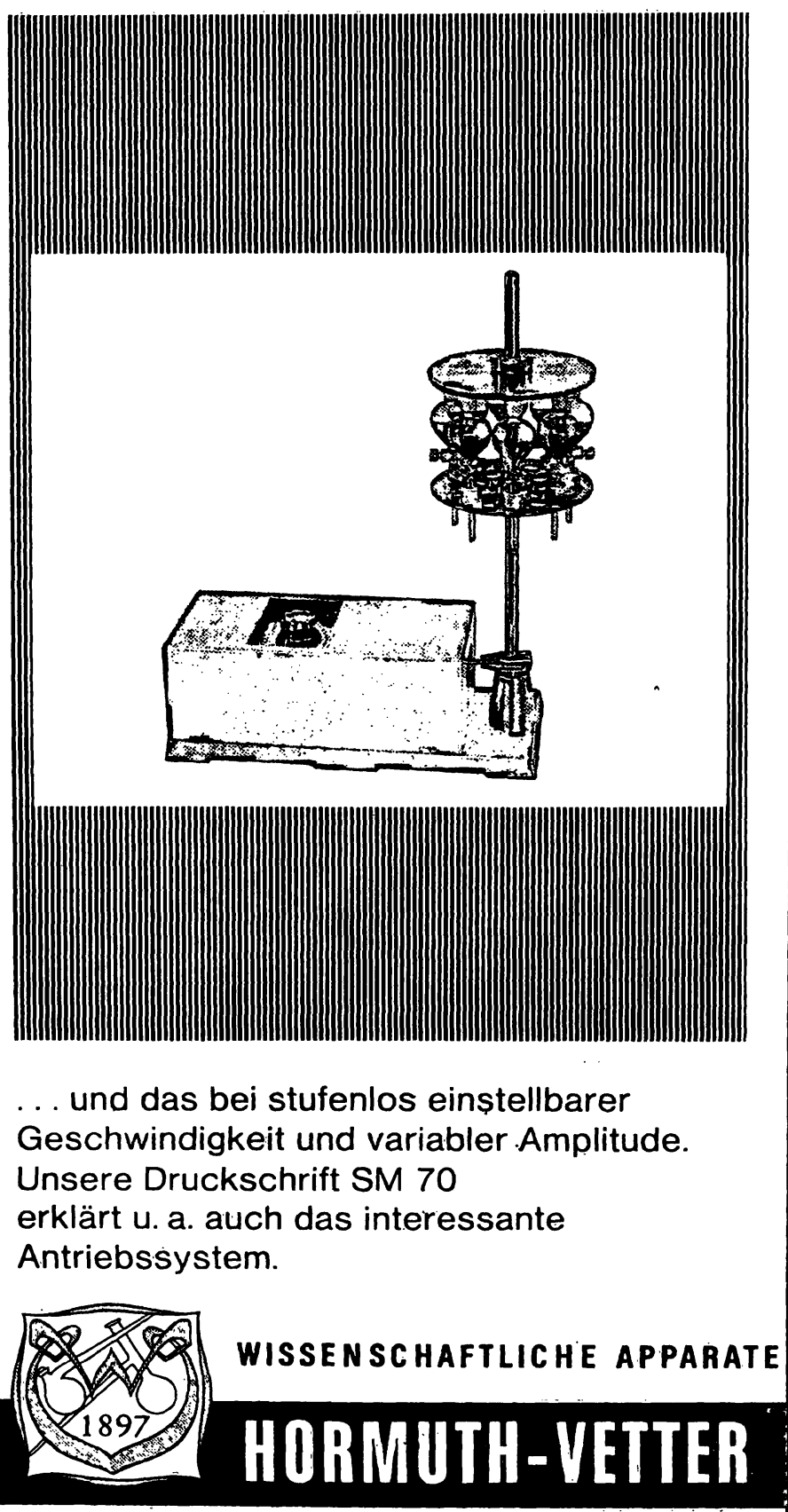

6908 Wiesloch/Bd., Postfach 1348, Tel. 0.6222/21 47 6900 Heidelberg 1, Postfach 750, Tel. 0622120045 


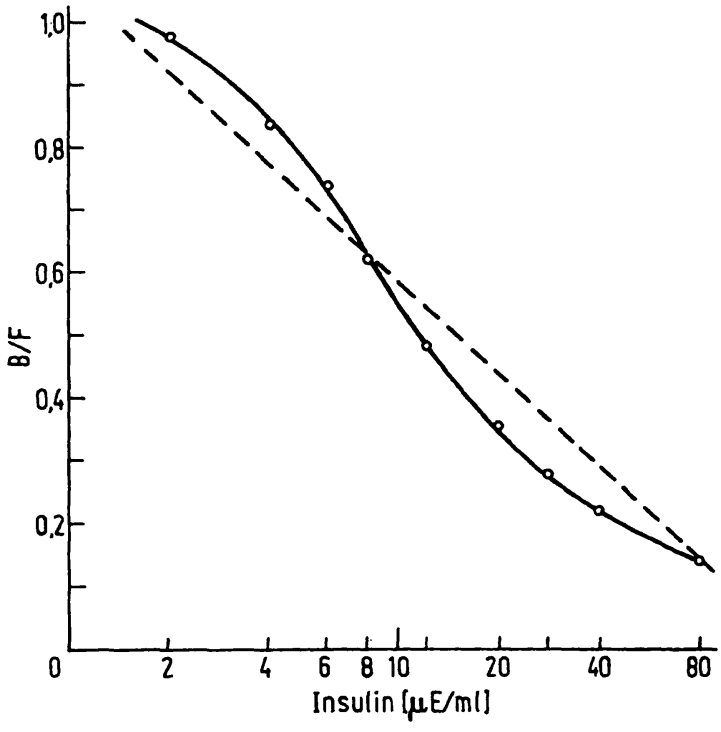

Abb. 3

Die Standardkurve halblogarithmisch aufgetragen Ordinate: B/F linear, Abszisse: Insulinkonzentration (logarithmisch)

daß es sich bei der vorliegenden Standardkurve um eine hyperbole Funktion handelt. Von der Theorie her ist mit einem solchen Verlauf dann zu rechnen, wenn der Assay sich im Zustand der Sättigung oder in Nähe der Sättigung befindet. In einem solchen Fall böte sich eine „Logit-Transformation" an, wie sie RoDBARD und Mitarbeiter (31) vorgeschlagen, und wie Abbildung 4 zeigt, kann dadurch auch eine gute Linearisierung erreicht werden.

Diese Methode zur Linearisierung liefert auch eine günstige Möglichkeit zur graphischen Interpolation. Eine andere einfache Methode, die auf BuIss (32) zurückgeht, kommt zur linearen Transformation durch Modifikation der X-Variablen. Die Größe, mit der die Variable X modifiziert wird, läßt sich in erster Näherung aus der Abbildung 4 gewinnen. Durch graphische Interpolation bei dem Y-Wert für $50 \%$ bzw. Logit $Y=0$, wird der zugehörige $X$-Wert aufgesucht, der dann approximativ die Größe c liefert. Die Größe c beträgt in unserem Fall $c=21$.

Abbildung 5 zeigt die erhaltene lineare Transformation, die zur Auswertung der Meßergebnisse herangezogen werden kann.

Eine ausführliche Würdigung und nähere Beschreibung dieser Auftragstechniken nach algebraischen wie statistischen Gesichtspunkten findet sich in der schon erwähnten Arbeit von RodBARD und Mitarbeitern (31). Wie man sieht, eignen sich die vorgeschlagenen Verfahren gut dazu, den vorliegenden Radioimmunoassay zu linearisieren und gestatten damit die angestrebte schnelle und exakte Auswertung. Allerdings sollte dazu ein elektronischer 'Tischrechner vorhanden sein. Von Hand ist die Kalkulation mühselig.

Für die technische Assistenz sei an dieser Stelle Frau H. Konter gedankt.

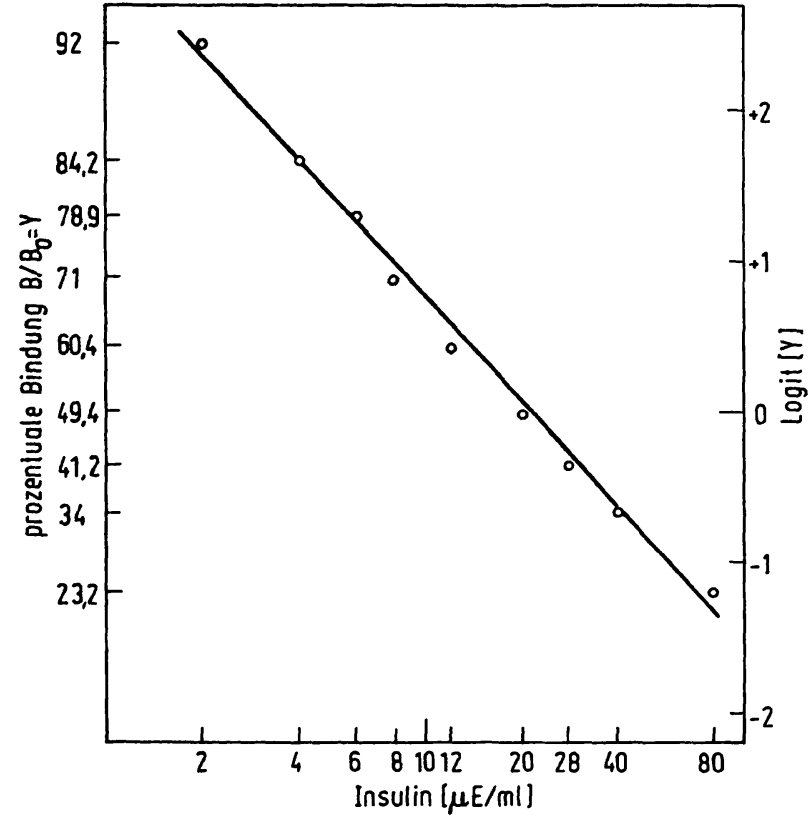

Abb. 4

Die Standardkurve in einer Logit-Transformation. Die abhängige Variable wird folgendermaßen umgeformt:

$$
\operatorname{logit}(Y)=\log _{e}\left[\frac{Y}{100-Y}\right] \text {, wobei } Y=B / B_{0} \text { ist. }
$$

Der erhaltene Wert wird auf der Logit-Skala (rechts) aufgetragen. Dazu korrespondierend findet sich links die Prozent-Skala. Abszisse: Insulinkonzentration in $\mu \mathrm{E} / \mathrm{ml}$ auf einer logarithmischen Skala. Diese Logit-log-Auftragung vermag den s-förmigen Kurvenverlauf in Abbildung $3 \mathrm{zu}$ linearisieren

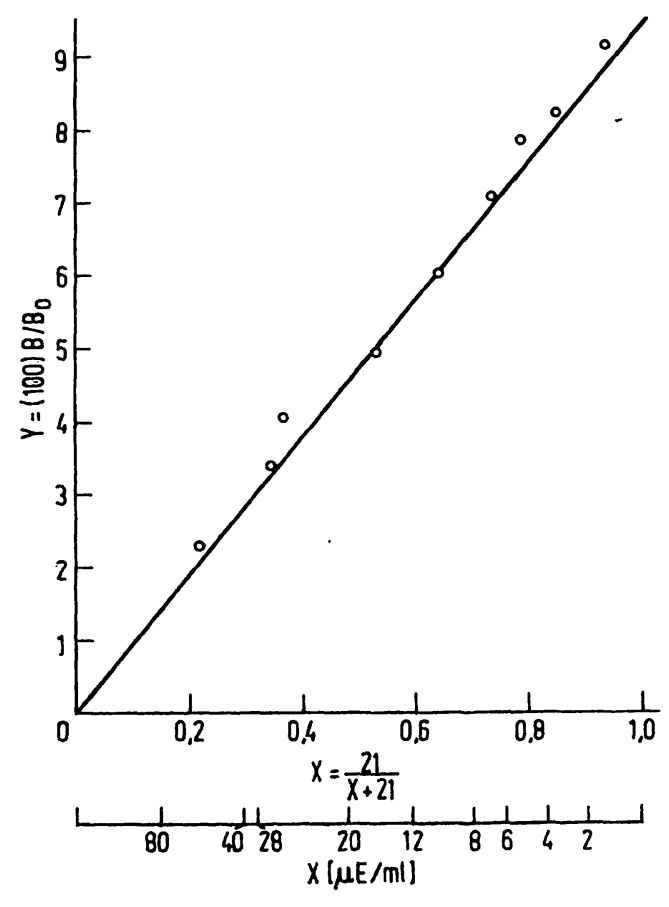

Abb. 5

Die Standardkurve linearisiert nach einem Vorschlag von Bliss (32) Ordinate: $Y=(100) B / B_{0}$.

Abszisse: Umgeformte $X$-Variable, $X^{\prime}=\frac{c}{X+c}$, wobei $c$ approximativ aus den $50 \%$ (bzw. Logit $Y=0$ )-Wert in Abbildung 4 bestimnt werden kann. Die zweite Abszissenskala zeigt die korrespondierenden $\mathrm{X}$-Werte (Insulinkonzentration $\mu \mathrm{E} / \mathrm{ml}$ ) 


\section{Literatur}

1. YALOw, R. S. und S. A. Berson, J. clin. Invest. 39, 1157 (1960). 2. Melani, F., H. Ditschunetr, K. M. Bartel, H. Friedrich und E. F. Pfeiffer, Klin. Wschr. 43, 1000 (1965). - 3. Skom, J. H. und D. W. Tulmage, J. clin. Invest. 37, 783 (1958). 4. Grodsky, G. M. und P. H. Forsham, J. clin. Invest. 39, 1070 (1960). - 5. Hales, C. N. und P. J. RaNDLE, Biochem. J. 88 , 137 (1963). - 6. Morgan, C. R. und A. Lazarow, Diabetes, N. Y. 12, 115 (1963). - 7. Herbert, V., K.-S. LaU, C. W. Gotrtuieb und S. J. Bleicher, J. Clin. Endocr. Springfield, 25, 1375 (1965). 8. Meade, R. C. und H. M. KlrtgaARd, J. Nucl. Med. 3, 407 (1962). - 9. WIDE, L. und J. PORATH, Biochim. biphysica Acta (Amsterdam), 130, 257 (1966). - 10. Berson, S. A. und R. S. YALOW, J. clin. Invest. 38, 1996 (1959). - 11. Karser, H. und H. SpeCKer, Z. analyt. Chem. 149, 46 (1956). - 12. Mrtcheld, M. L. und J. Byron, Diabetes, N. Y. 16, 656 (1957). - 13. Mrtchell, M. L., S. Collins und J. Byron, J. clin. Endocr. 29, 257 (1969). - 14. Yalow, R. S. und S. A. Berson, J. clin. Invest. 36, 648 (1957). - 15. Williams, R. H., Metabolism, Baltimore, 5, 128 (1956). - 16. Nomoto, M., Y. Narafiashi und M. MURAKami, J. Biochem. 48, 593, 906 (1960). - 17. Hashimoto, Y., S. Tsuiki, H. Nisizawa und W. Pigman, Ann. N. Y. Acad. Sc. 106, 233 (1963). - 18. Yalow, R. S., und S. A. Berson, Meth. Biochem. Analysis, 12, 69 (1964). - 19. CatT, K. J., H. D. Nrall und G. W. Tregear, Biochem. J. 100, 31c (1966). -
20. Catt, K. J. und G. W. Tregear, Science 158, 1570 (1967). 21. Ceska, M., F. GrossmülLER und U. LUNDKwist, Acta endocr., K'hvn. 64, 111 (1970). - 22. Pincus, G., K. V. Thrman und E. B. Asrwood, The Hormons, Vol. IV, Academie Press London, New York (1964). - 23. EkrNs, R. P., Theoretical aspects of saturation analysis in Acta Endorcr., Supplement 147 (1970). 24. Poots, J. T. Jr., L. M. SHERwood, J. L. H. O'Riordan und G. D. Aurbach, Advances Int. Med., N. Y. XIII, 185 (1967). 25. Ekins, R. P., G. B. NewManN und J. L. H. O RIORDAN, in „Radioisotopes in medicine: In vitro studies „Theoretical aspects of saturation and radioimmunoassay. Editors: Hayes, R. L., Goswitz, F. A., und Murphy, B. E. P., Oak Ridge, Tenn. 1968 United States Atomic Commission pp. 59-100. - 26. Hunter, W. M., „Protein and Polypeptide Hormones" Proceedings of the International Symposium, Liege (1968), M. Margoulies Excerpta Medica Foundation Amsterdam (1969). - 27. Hunter, W. M. und F. C. GreENwood, Biochem. J. 91,43 (1964). - 28. QuABBE, H.-J., Diabetologia 5, 101 (1969). - 29. Gaddum, J. H. Pharmacol. Rev., Baltimore, 5, 87 (1953). - 30. GADDUM, J. H., J. Pharmacy Pharmacol., London 6, 345 (1953). - 31. RodBard, D., W. Bridson und P. L. RAYFord, J. Lab. Clin. Med. S. Louis, 74, 770 (1969). - 32. Blrss, C. L., Proceedings of the workshop conference on Statistics in Endocrinology, Dedham, Mass., Cambridge, Massachusetts Institute of Technology Press. In press.

Dr. rer. nat. Helmut Wombacher Abt. Klin. Endokrinologie II. Med. Klinik, Uni. Mainz 6500 Mainz 